\title{
ACUTE BILATERAL SUPRARENAL HAEMORRHAGE
}

\author{
BY \\ GAVIN C. ARNEIL, M.B., Ch.B. \\ (From the Royal Hospital for Sick Children, Glasgow, and the Department of \\ Medical Paediatrics, University of Glasgow)
}

Acute bilateral suprarenal haemorrhage after infection has come to be recognized as a clinical entity only in the past fifty years. The first report on the subject was published in 1894 by Voelcker, who described the pathology of a case. This was followed, in 1898 , by reports on one case each by Batten, Andrewes, and Garrod and Drysdale. The first review of the clinical findings as seen in five cases, was given by Little in 1901. Andrewes, in 1906, was the first to isolate $\mathbf{N}$. meningitidis in this condition. Altogether seventeen cases had been reported in the British literature before Dudgeon published the first case in America in 1904. In 1911 Waterhouse reviewed fourteen cases and added one more. Friderichsen then published two cases in 1918.

In recent years the literature has been reviewed on many occasions, notably by Aegerter in 1936, Sacks in 1937, Lindsay, Rice, Selinger, and Robins in 1941 and by Kundstadter in 1939. In 1944 Bernhard and Jordan raised the total of cases published to one hundred and seventeen, of which eighteen were in adults. Since then, twenty-eight more cases have been reported, which, with the ten cases described herein, brings the total to one hundred and fifty-five; in addition 'cures' have been claimed on ten occasions.

Acute bilateral suprarenal haemorrhage is precipitated by a sudden septicaemia, usually meningococcal, resulting in destruction by haemorrhage of the medulla of both suprarenals. In most cases the patient is under two years of age, and the sexes are equally affected. The usual history is of a previously healthy child, aged six to eight months, awakening one morning cross or irritable and refusing his feeds. Diarrhoea and vomiting ensue. The respiratory rate increases, and the breathing becomes acidotic or Cheyne-Stokes in type. The temperature is usually raised, and the picture is suggestive of bronchopneumonia but without chest signs. This may be described as the stage of pseudopneumonia. The pulse becomes very weak and rapid and is sometimes irregular. Older children complain of vague abdominal pains and headache. Drowsiness and coma rapidly supervene. The child is cyanosed and presents the picture of extreme circulatory collapse, the weak rapid irregular pulse being a marked feature. There frequently appears a patchy, purple mottling of the skin which resembles postmortem lividity. At this point, about eight to nine hours from the onset of illness, the child may die or may develop a diffuse purpuric eruption. This may consist of fine ecchymoses or patches of intradermal haemorrhage, up to five centimetres in diameter (Kundstadter, 1939). The blood pressure is extremely low, and bilateral basal râles-a sign of developing pulmonary oedema-are heard in most cases. At this stage, because of the presence of skin manifestations, the appearance of the patient differs from that seen in bronchopneumonia. Death occurs thirty minutes to forty hours after the appearance of the rash.

Banks and McCartney (1942) divide their cases into two types: (a) the pure adrenal type, showing comparative clarity of intellect, with acidotic breathing; (b) the encephalo-adrenal type, showing early coma with stertorous or Cheyne-Stokes breathing. In both types the other signs and the post-mortem findings are similar, apart from the presence of early encephalitis in type (b).

\section{Case Reports}

The following ten cases have been taken from the records of the Royal Hospital for Sick Children, Glasgow. Two of these cases (V. A. and I. G.) came under the personal observation of the author.

Case 1. G. Q., a female infant aged two weeks, was born by normal delivery, and was well until twenty-four hours before admission, when the respiratory rate increased. Six hours before admission, widespread purpuric spots appeared, and the breathing became stertorous; the child died one hour after admission. On examination there was a diffuse purpuric rash and a large intradermal haemorrhage in the left axilla; the respiratory rate was rapid, and fine râles were present at both bases; the spleen and liver were palpable; Kernig's sign was negative; there was no nuchal rigidity. The duration of the illness from onset till death was twenty-five hours.

At autopsy, there was a widespread purpuric eruption, a small bilateral pleural exudate, and petechiae on the pleura; the thymus was not enlarged. There was congestion of the spleen, swelling of the mesenteric glands, and complete bilateral suprarenal haemorrhage. The brain and meninges were normal. The bone marrow, spleen, and pleural fluid were sterile on aerobic culture.

Case 2. B. L., a male, seven weeks old, was born by Caesarian section. He was perfectly well until twelve hours before admission, when deep, rapid breathing began. For eight hours before admission there was also diarrhoea. On examination the temperature was $100^{\circ} \mathrm{F}$., there was no cyanosis, 
breathing was rapid and deep, the pulse rapid and weak, and the abdomen normal. There was no nuchal rigidity; Kernig's sign was negative. The child developed deep cyanotic patches and purpura after eleven hours. The duration from onset till death was twenty-seven hours.

At autopsy there was a diffuse purpuric rash. . The thymus and thoracic glands were not enlarged. There was slight congestion of the lung bases. The mesenteric glands, liver, and kidneys appeared normal. There was congestion of the spleen and gastro-intestinal tract, and complete bilateral suprarenal haemorrhage. Meninges appeared normal.

Case 3. O. G., a male, aged eight months, ten hours before admission became drowsy, passed four loose stools, and began breathing rapidly. On examination the temperature was $104^{\circ} \mathrm{F}$., and the respirations 60 per minute. The child was pale and drowsy, with no cyanosis but a rather mottled appearance of the skin. Breath sounds, heart sounds, and abdomen were normal. Kernig's sign was negative. There was no nuchal rigidity. The cerebrospinal fluid was clear, the pressure high, the globulin content normal, two cells per c.mm., culture negative. A petechial rash appeared on the face and trunk eleven hours after onset. The duration from onset till death was thirteen hours.

At autopsy the child was cyanosed, with a petechial rash and a large thymus. There was some oedema of lung bases. The heart was normal. The mesenteric glands were swollen, and the liver and spleen congested. Occasional small submucous haemorrhages were found in the ileum. There was complete bilateral suprarenal haemorrhage, and some oedema of brain. Culture of the spleen and heart blood showed a growth of enterococci (regarded as contaminant).

Case 4. L. B., a female, aged eight months, seven hours before admission vomited and refused feeds. Five hours later respirations became rapid. Ten minutes before admission a purpuric eruption appeared. On examination the child was dyspnoeic and rather cyanosed; she was covered with purpuric blotches. The lungs appeared normal; the pulse was weak and irregular. Kernig's sign was negative. There was no nuchal rigidity. The cerebrospinal fluid was clear, pressure and globulin normal, three cells per c.mm.; culture yielded a growth of $\mathbf{N}$. meningitidis. Blood culture, N. meningitidis. The duration from onset till death was twelve hours.

At autopsy there was a diffuse purpuric eruption and a large thymus. Heart, lungs, and pleurae appeared normal; the mesenteric glands and spleen were large, and the ileal contents mucoid. There was complete bilateral suprarenal haemorrhage; brain and meninges appeared normal. Blood culture taken at autopsy yielded only a growth of enterococci (regarded as contaminant).

Case 5. C. L., a male, was aged two years. Eighteen hours before admission he woke, became restless, vomited, and passed three loose stools; ten hours before admission widespread purpura appeared over the trunk and limbs. On examination the temperature was $101^{\circ} \mathrm{F}$., there were widespread petechiae and a purpuric rash on the skin; some rhonchi were heard in the right chest; the pulse was weak and rapid, but regular. Kernig's sign was negative, and there was no nuchal rigidity. Louco- cytes were 23,000 per c.mm. of blood, and platelets 136,000 per c.mm. Cerebrospinal fluid pressure was raised; there was no increase in globulin, 7 cells per c.mm. ; culture was sterile. Duration from onset till death was fifty-two hours.

At autopsy there was a diffuse purpura of limbs and trunk; lungs, pleura, and heart appeared normal; there were scattered epicardial petechial haemorrhages; the spleen was enlarged, and there was complete bilateral suprarenal haemorrhage; the brain and meninges appeared normal. The cerebrospinal fluid, spleen, and blood were sterile on culture.

Case 6. V. A., a male, aged three years, was cross, irritable, and breathing rapidly for twelve hours. Six hours before admission he developed a blotchy, purpuric rash and became comatose. The child was dead on admission, the body being deeply cyanosed, with purpura of the trunk. The duration from onset till death was fourteen hours.

At autopsy a well-nourished body was found, with large purpuric blotches and fine petechiae over the skin. The thymus was not enlarged; lungs, pleura, and heart appeared normal; there was congestion of the liver and spleen; the mesenteric glands were large; Peyer's patches were enlarged, and the ileal contents mucoid. There was complete bilateral suprarenal haemorrhage, and also slight congestion and oedema of brain. On culture of spinal fluid, N. meningitidis was recovered.

Case 7. I. G., a female, aged four months, was perfectly well until ten hours before admission, when she refused her feed, moaned, and began rapid, deep breathing. Two loose brown stools were passed. On examination the temperature was $102^{\circ} \mathrm{F}$., the child was cyanosed, the buttocks purple, and there was rapid, harsh, grunting breathing ( 50 per minute) with râles at the bases of the lungs; the pulse was rapid and very weak. Kernig's sign was negative; there was no nuchal rigidity. Leucocytes were 15,000 per c.mm. of blood. The duration from onset till death was ten hours.

At autopsy the body was plump and cyanosed, there was congestion and marked hypostasis of the skin, the thymus was not enlarged, the heart and pleura appeared normal, there was oedema of the lung bases, the mesenteric glands were enlarged, the gastro-intestinal tract was congested and there was complete bilateral suprarenal destruction. The meninges were rather congested. Cultures post mortem yielded only coliform bacilli.

Case 8. S. R., a male, aged seven months, ten hours before admission vomited and passed several loose yellow stools. Respiratory rate was rapid for eight hours. On examination the child was cyanosed, the temperature $101^{\circ} \mathrm{F}$., the respiration shallow and rapid (45 per minute), and the pulse weak and irregular. Kernig's sign was negative. There was no nuchal rigidity. The duration from onset till death was ten hours.

At autopsy the thymus was large, there was congestion and oedema of the lung bases, the heart was normal, the mesenteric glands and spleen enlarged, the colon inflamed. Peyer's patches were enlarged, the ileal contents fluid and green, and there was complete bilateral suprarenal haemorrhage. No abnormality was observed in the brain. Cultures taken post mortem yielded only coliform bacilli. 
Case 9. P. R., aged two years seven months, six hours before admission vomited, and passed a loose stool containing blood and mucus. Three hours before admission he became pale and comatose, and he died on arrival at the hospital. The duration from onset till death was six hours.

At autopsy the body was found to be wellnourished, the skin was normal, and the thymus small. There was oedema of the lung bases. Tuberculous mesenteric glands were found. Peyer's patches were enlarged, and the gastro-intestinal tract congested; there was complete bilateral suprarenal haemorrhage, and cerebral oedema, but there was no evidence of meningitis. Culture of the ileal contents, spleen, and Peyer's patches yielded growth of coliform bacilli.

Case 10. G. L., a male, aged three years, five hours before admission awoke and passed a green stool. One hour before admission he vomited, became drowsy, and passed four loose stools. He then became pale, and the respiratory rate increased. On examination the temperature was $101^{\circ} \mathrm{F}$. Respirations were shallow ( 40 per minute). He was very collapsed and cyanosed. There were no abnormal physical signs in the chest; the pulse was rapid and weak. The abdomen was normal, Kernig's sign was negative, and there was no nuchal rigidity. The stool contained blood, mucus, and pus. The child became unconscious and cyanosed, and died two hours later. The duration from onset till death was seven hours.

At autopsy the thymus and thoracic glands were enlarged. Lungs and heart appeared normal, the mesenteric glands were enlarged, no abnormality of the spleen, liver, and kidneys was noted, there was marked ileocolitis, and complete bilateral suprarenal haemorrhage. Brain and meninges were normal.

The ages of these ten children ranged between two weeks and three years, no less than four being over the age of two years. Epidemiologically, it is interesting to note that, in 1943, three cases occurred within five days of each other, and, in 1945, two cases within twenty-four hours. On neither occasion was any connexion between the cases apparent. Similar observations have been recorded (Garrod, Batten, and Thursfield, 1934).

The table shows the frequency with which the most common clinical and pathological features appeared in the series under discussion. The cases have been divided, for comparison, into those with purpura and those without.

Most frequently the child arrived in hospital at the stage of pseudopneumonia. There were no signs of meningeal irritation, and the only features inconsistent with a diagnosis of bronchopneumonia were the presence of the rash and the absence of physical signs of disease in the lungs. The average temperature was about $101^{\circ} \mathrm{F}$. No relationship was apparent between the temperature and the degree of suprarenal destruction. Lumbar puncture was done in three cases, and revealed a clear fluid, a rise in pressure, and a cell count of two to seven cells per cubic millimetre. The blood leucocyte count was raised. Post-mortem examination revealed in each case complete obliteration of the
TABLE 1

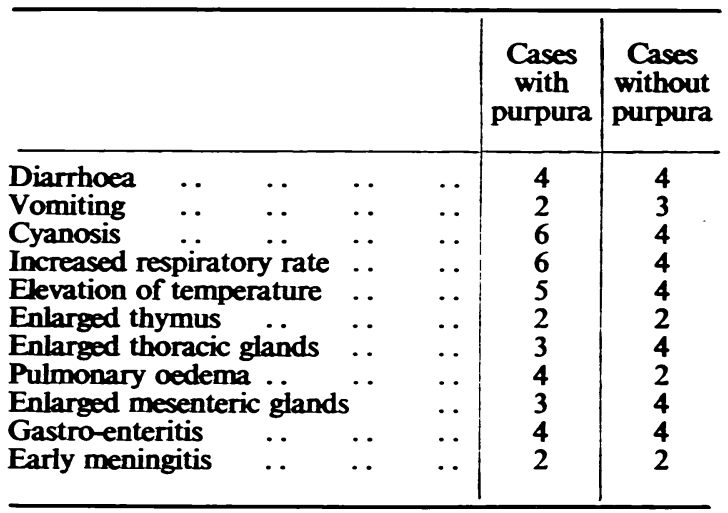

medullary tissue of both suprarenals, with a varying degree of cortical damage. In none of these cases was the capsule ruptured. Microscopically, a gross infiltration of all medullary tissue by red blood cells with destruction of the secreting tissue was seen. Another common finding, more marked in the fulminating cases, was enlargement of the thoracic and mesenteric lymph glands, which may be attributed to the septicaemia. This fact was noted by Montford and Mehrling (1941), and by Bernhard and Jordan (1944). Inflammation of the gastrointestinal tract was noted in 80 per cent. of cases, a figure which closely corresponds to the number of cases giving a history of diarrhoea. In two cases hypertrophied Peyer's patches were noted. In four of the ten cases there was an unusually large thymus.

The cases have been divided into two types: (a) those cases with purpura, and (b) those cases without purpura. The classical cases quoted in the literature have always included purpura as an important sign. In the table it is seen that the clinical and pathological findings in the two groups are not very different. In every case the existence of bilateral suprarenal haemorrhage of acute onset was proved by pathological examination. Generally speaking those cases without purpura were more fulminating, and showed less leucocytic reaction and more signs of a severe toxic condition than the purpuric cases. The most significant difference between the two groups, however, lay in their duration. The non-purpuric cases survived, on the average, only eight hours after the onset of symptoms, while the purpuric cases, on the average, developed their rash ten and a half hours after the onset of illness, and died in twenty-four hours.

In view of this, it seems reasonable to suggest that non-purpuric cases are etiologically similar to those showing purpura, but that the infection in the former type is more overwhelming and the patient succumbs before the purpura appears. The cause of this catastrophe is now widely accepted to be a septicaemia, with resulting presence of exotoxins in the blood, and with consequent damage to the weakly supported vessels of the suprarenal medulla, producing destruction of suprarenal tissue by haemorrhage. Other explanations have been advanced, 
such as thrombosis of suprarenal veins (Firor, 1937), direct bacterial embolization, as proved by direct suprarenal culture, and 'thrombotic necrosis or focal adrenalitis' (Banks and McCartney, 1942). Keele and Keele (1942), describing ten cases of suprarenal infarct in adults, were careful to differentiate this type of case from those described above. The most commonly accepted view of the cause, namely, that there is a toxic action, is given added weight by the work of Olitzki (1942), who demonstrated the production of bilateral suprarenal haemorrhage in guinea-pigs by the injection of various organisms such as $\mathbf{N}$. meningitidis and H. influenzae. Also, bilateral suprarenal haemorrhage in guinea-pigs after infection with diphtheria bacilli or toxin is a finding which forms the basis of a test of the virulence of the organisms.

In 1944 Marangoni and D'Agati (1944) published two cases which, under intensive therapy, survived the suprarenal haemorrhage for eighty hours and died from the 'hepato-renal syndrome.' They infer from this that the suprarenals concentrate and detoxicate the toxins, and that after their destruction the toxins act on liver and kidney tissue.

With regard to the actual invading organisms, it is significant that in 70 per cent. of published cases $\mathbf{N}$. meningitidis has been recovered. In the remaining cases, where this organism was not recovered, it is important to remember that, though intra-vitam cultures were done in a few, in the majority this was not done and no culture was made until many hours after death. It is, of course, well known that meningococci are very delicate and can only be recovered relatively soon after death. Morrison (1943) considers the time limit to be twelve hours; in case 4, intra-vitam culture grew $\mathbf{N}$. meningitidis, whereas culture twelve hours post mortem did not. A point of interest is the work of McLean and Caffey (1931), who, by staining smears and culturing the contents of purpuric blebs, isolated the meningococcus in 83 per cent. of cases. There still remain some cases in which blood culture during life has proved negative, or in which another organism has been isolated, as in Lindsay's case (1941), where $H$. influenzae was found. It seems reasonable, therefore, to postulate that in a few cases the haemorrhage is produced in a similar fashion, but by toxins of organisms other than $\mathbf{N}$. meningitidis. This is confirmed by the work of Olitzki (1942).

It can be accepted that the total destruction of both suprarenal glands without some adequate or sustained hormone therapy is quite incompatible with the continuance of life. That chemotherapy might overcome meningococcal septicaemia is a reasonable claim, even when concomitant coma or purpuric rash is present; but to claim that a short course of penicillin or sulphonamide will restore function to, or replace the function of, a vital hormone-producing organ which has been destroyed, seems an unwarranted and untenable presumption. Such cases of bilateral suprarenal haemorrhage recorded as cures may be examples of fulminating meningitis with purpuric eruption (spotted fever), or of meningococcal encephalitis with concomitant rash, in which little or probably no suprarenal damage has occurred. While intensive chemotherapy might be used in an attempt to check this fulminating disease, it must be obvious to all that, long before diagnosis is possible, irreparable damage would have been done to vital suprarenal tissue and there would be little chance of preventive treatment being successful. Even if success were achieved in dealing with the acute stage, complete hormonal replacement would be required to preserve life.

In recent years the name Waterhouse-Friderichsen syndrome has come to be applied to this condition. Holmes and Cowan (1945) have raised objections to this. Not only is the name without historical significance, but it has led to various misinterpretations of the actual syndrome, and does nothing to indicate the essential feature of suprarenal damage. The name ' acute bilateral suprarenal haemorrhage, which is self-explanatory, accurate, and specific, is suggested.

\section{Summary}

Ten cases of acute bilateral suprarenal haemorrhage have been described. The disease is consistently fatal, many cases dying before the purpuric rash has developed. The most frequently recovered invading organism is the $\mathbf{N}$. meningitidis.

The author's thanks are due to Dr. Stanley Graham for his encouragement in preparing this paper, to Professor G. B. Fleming for permission to publish four of the case reports, and to Dr. George Montgomery for the autopsy reports.

\section{REFERENCES}

Aegerter, E. E. (1936). J. Amer. med. Ass., 106, 1715. Andrewes, F. W. (1898). Trans. path. Soc., London, 49, 259.

Banks, H. S., and McCartney, J. E. (1942). Lancet, 1, 219.

Batten, F. E. (1898). Trans. path. Soc., London, 49, 258. Bernhard, W. G., and Jordan, A. C. (1944). J. Lab. clin. Med., 29, 357.

Dudgeon, L. S. (1904). Amer. J. med. Sci., 127, 134.

Firor, W. M. (1937). Southern med. J., 30, 306.

Friderichsen, C. (1918). Jb. Kinderheilk., 87, 109.

Garrod, A. E., and Drysdale, J. H. (1898). Trans. path. Soc., London, 49, 257.

_- Batten, F. E., and Thursfield, H. (1934). Diseases of Children, London.

Holmes, J. M., and Cowan, J. M. (1945). Lancet, 1, 13.

Humphrey, A. A., and Toomey, L. O. (1944). Nav. med. Bull., Wash., 42, 165.

Keele, D. V., Keele, K. D. (1942). Brit. med. J., 2, 687.

Kunstadter, R. H. (1939). Arch. Pediat., 56, 489.

Lindsay, J. W., Rice, E. C., Selinger, M. A., and Robins, L. (1941). Amer. J. med. Sci., 201, 263.

Little, E. G. (1901). Brit. J. Derm. Syph., 13, 445.

McLean, S., and Caffey, J. (1931). Amer. J. Dis. Child., 42, 1053 .

Marangoni, B. A., D'Agati, V. C. (1944). Amer. J. med. Sci., 207, 385.

Monfort, J. A., and Mehrling, J. H. (1941). Amer. J. Dis. Child., 62, 144.

Morrison, J. E. (1943). Lancet, 1, 800.

Olitzki, L. et al. (1942). J. Immunol., 45, 237.

Sacks, M. S. (1937). Ann. intern. Med., 10, 1105.

Voelcker, A. F. (1894). Path. Reports of Middlesex Hosp. (Quoted by Waterhouse.)

Waterhouse, R. (1911). Lancet, 1, 577. 